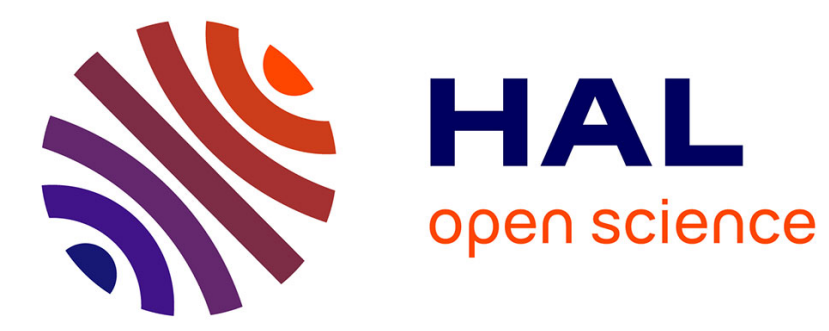

\title{
Du bon usage des politiques monétaire et budgétaire en Europe
}

Jérôme Creel, Henri Sterdyniak

\section{To cite this version:}

Jérôme Creel, Henri Sterdyniak. Du bon usage des politiques monétaire et budgétaire en Europe. Revue d'économie financière, 1998, 45, pp.167-196. 10.3406/ecofi.1998.2500 . hal-01010410

\section{HAL Id: hal-01010410 \\ https://hal-sciencespo.archives-ouvertes.fr/hal-01010410}

Submitted on 19 Jun 2014

HAL is a multi-disciplinary open access archive for the deposit and dissemination of scientific research documents, whether they are published or not. The documents may come from teaching and research institutions in France or abroad, or from public or private research centers.
L'archive ouverte pluridisciplinaire HAL, est destinée au dépôt et à la diffusion de documents scientifiques de niveau recherche, publiés ou non, émanant des établissements d'enseignement et de recherche français ou étrangers, des laboratoires publics ou privés.

\section{(이) $\$$}

Distributed under a Creative Commons Attribution - NonCommercial - NoDerivatives| 4.0 


\section{DU BON USAGE DES POLITIQUES MONÉTAIRE ET BUDGÉTAIRE EN EUROPE}

JÉROME CREEL ET HENRI STERDYNIAK*

$\mathrm{I}$

L EST MAINTENANT pratiquement certain qu'une monnaie unique sera mise en place en Europe en 1999 et que l'UEM regroupera la quasi-totalité des pays de l'UE (soir 11 sur quinze, le RoyaumeUni, le Danemark, la Suède ayant choisi de rester à l'écart et la Grèce r'étanr de toute évidence pas prête). Reste que des questions délicates quant à l'organisation des politiques économiques n'ont pas été résolues : comment rendre compatible une politique monétaire unique avec des conjonctures spécifiques, des politiques budgétaires autonomes, des marchés du travail différenciés, des structures économiques différentes? Le traité de Maastricht, puis le Pacte de stabilité, ont dessiné un type d'organisation précis, mais dont la viabilité n'est pas assurée : une Banque centrale européenne (BCE) indépendante ayant essentiellement un objectif de lutte contre l'inflation, des limites aux déficirs publics sans coordination organisée des politiques budgétaires et sans stratégie coordonnée entre la politique monétaire er les politiques budgétaires. En cas de choc généralisé en Europe, quel sera le partage des rôles entre la BCE et les autorités budgétaires? Faut-il l'organiser ou faut-il espérer que des comportements autonomes des autorirés monétaires et budgétaires aboutiront spontanément à une combinaison efficace? En cas de choc spécifique, le pays touché devaait pouvoir pratiquer une politique budgétaire d'autant plus active qu'il ne pourra compter sur la politique monétaire. Le Pacte de stabilité vise à rassurer ceux qui craignaient que des politiques budgétaires autonomes induisent un biais inflationniste et expansionniste, qui obligerait la BCE à maintenir des taux d'intérêt trop élevés. Mais, la contrainte ainsi créée ne risque-t-elle pas d'empêcher les politiques budgétaires de jouer leurs rôles? De plus, la mise en place de l'UEM survient dans une période difficile : si l'inflation est vaincue, l'Europe souffre d'une croissance trop lente, de la persistance du chômage de masse et d'une hausse tendancielle de ses dettes publiques. Entre la BCE, la Commission, le Conseil et les États, comment une stratégie économique plus efficace pourrait être définie et mise en ouvvre? Par ailleurs, la globalisation financière impose de nouvelles contraintes:

* Respecrivement : CREFED, universiré de Paris DX-Dauphine ex OFCE. 
une politique économique reste-t-elle possible ou les gouvernements doivent-ils se plier à la volonté des marchés financiers?

Deux stratégies macroéconomiques s'opposent en Europe. Pour les uns, l'UEM sera l'occasion de recréer un vaste espace relativement fermé où la politique économique pourra retrouver son efficacité : la contrainte extérieure sera limitée, les problèmes d'inefficacité des politiques non-coordonnées disparaitront. L'Europe pourra mieux protéger son modèle social. La puissance de l'euro permettra de pratiquer de bas taux d'intérêt et de faire baisser le taux de change de la monnaie européenne. Ceci suppose un gouvernement économique européen, capable de dialoguer avec la BCE et d'organiser les politiques budgétaires. Mais, ce gouvernement n'est pas inscrit dans le traité; son existence pose de délicats problèmes de souveraineté. Rien ne garantit que la BCE pratiquera effectivement une stratégie de bas taux d'intérêt et de bas niveau de l'euro. Pour les autres, l'Europe souffre avant tout de trop de réglementations, de trop de dépenses publiques et de trop d'impôts, en particulier de charges sociales trop élevées. L'UEM doit être l'occasion d'une nouvelle pratique de la politique économique, capable de séduire les marchés financiers. Il importe donc d'assurer l'indépendance de la $B C E$, de pratiquer une politique d'euro fort, de lier les mains aux poliriques budgétaires. Chaque pays devra avant tout améliorer ses conditions d'offre en réduisant ses dépenses publiques et ses charges. Dans ce contexte, la coordination des politiques budgétaires pourrait être dangereuse, en incitant les pays à pratiquer des politiques expansionnistes, inflationnistes et déséquilibrantes. La coexistence de ces deux points de vue contradictoires pèse lourdement sur les capacités de l'Europe à définir une politique économique active.

Cet article présente d'abord l'évolution des déficits et des dettes publics en Europe depuis 1990, période caractérisée par la coexistence paradoxale d'un fort chômage, de forts déficits publics et de hauts iniveaux des taux d'intérêt. Quels enseignements peut-on tirer de cet épisode douloureux? Faut-il y voir des politiques budgétaires trop expansionnistes, ce qui justifierait l'insistance mise dans le traité sur le contrôle des politiques budgétaires? Faut-il y voir l'effet de politiques monétaires trop restrictives, en situation de ralentissement économique? Pour répondre à ces questions, l'article analyse les liens entre, d'une part déficit public et taux d'intérêt, d'autre part entre politiques budgétaire et monétaire. Enfin, l'organisation des politiques monétaires et budgétaires en Union monétaire fait l'objet d'une comparaison avec la situation en SME ou en changes flexibles. Nous déterminons notamment les conditions dans lesquelles différents pays en situation de monnaie unique peuvent stabiliser leur niveau d'activité, en respectant les critères de finances publiques définis dans le Pacte de stabilité. 
DU BON USAGE DES POLITIQUES MONETAIRE ET BUDGETAIRE EN EUROPE

\section{LE CREUSEMENT DES DEFICITS ET DES DETTES PUBLICS EN EUROPE}

\section{Depuis les années soixante-dix, une politique budgétaire laxiste?}

Alors que ses Finances publiques étaient équilibrées dans les années soixante, l'Europe des 15 a vu ses déficits publics se creuser avec la récession du début des années soixante-dix. Le déficit public de l'UE à 15 est ainsi passé de $1,8 \%$ du PIB en 1972-1973 à 5,8\% en 1975 (graphique 1). Il s'est stabilisé à $4 \%$ environ entre 1976-1980. Après un nouveau creusement au début des années quatre-vingt, les déficits se sont réduits tendanciellement entre 1981 et 1989 jusqu'à $2,9 \%$ en 1989. Après 1990, le déficit s'accroît à nouveau fortement pour atteindre un maximum en 1993 (6,3\%) au creux de la récession européenne. Depuis 1993, les déficits publics européens se sont progressivement résorbés, tout en demeurant à un niveau élevé $(4,4 \%$ en moyenne en 1996) supérieur aux normes édictées par le traité de Maastricht.

L'accumulation des déficits des finances publiques a entrâné un net accroissement de la dette publique relativement au PIB (graphique 2). Le ratio dette/PIB reste stable entre 1970 et 1975 , commence à augmenter sous l'effet des déficits entre 1975 et 1980, mais c'est surtout à partir de 1980 que la dette augmente fortement, sous l'effet de la hausse des taux d'intérêt relativement au taux de croissance. De 1986 à 1990, la dette commence à diminuer en raison d'importants assainissements budgétaires et de la reprise d'une forte croissance. Paradoxalement, c'est en 1991, immédiatement après la signature du traité de Maastricht qui insrituait des critères de dette publique pour entrer dans l'Union économique et monétaire, que la dette nette connaît une nouvelle hausse : celle-ci atteint près de $60 \%$ du PIB en 1997. Cette hausse provoque une augmentation importante de la charge de la dette (de 1,8\% du PIB dans les années 1970 à 5,4 en 1996).

\section{Déficits et déficits structurels}

L'évolution des déficits et des dettes publics peut laisser l'impression d'un laxisme généralisé depuis 1973, laxisme renforcé même depuis 1990 et que la période récente tenterait de corriger. Deux éléments mettent en cause cette image :

- la dégradation de la conjoncture se traduit "naturellement " par des pertes de recetres fiscales et des transferts sociaux supplémentaires (stabilisateurs automatiques);

- la hausse des taux d'intérêt a alourdi la charge de la dette. 
Dans les deux cas (chute de la demande privée et hausse des taux d'intérêt), les exigences de stabilisation de la dette publique sont contradictoires avec celles de stabilisation de l'activité. Aussi la dérive des finances publiques ne correspond-elle pas toujours à des choix opérés par les autorités mais s'impose à elles en partie. Pour apprécier les impulsions délibérées données par la politique budgétaire, il faut calculer un solde structurel primaire, c'est-à-dire un solde public, hors charges d'intérêt de la dette, corrigé des écarts de la production à son potentiel (encadré 1).

Les années soixante-dix sont marquées par un consensus sur l'utilisation de la politique budgétaire pour lutter contre la stagnation économique; ceci se traduit par un fort creusement des déficits primaires structurels jusqu'à 3,5\% du PIB en 1975). Entre 1977 et 1980, les déficits publics se rapprochent de l'équilibre. À partir de 1982, le solde structurel primaire devient largement positif. Ce changement de cap provient de deux facteurs. D'une part, d'un revirement des objectifs : la lutte contre l'inflation domine celle contre le chômage dans les objectifs des autorités budgétaires et monétaires. D'autre part, le fort niveau des taux d'intérêt oblige les autorités budgétaires à réduire les déficits primaires. Les déficits structurels se stabilisent entre 1987 et 1989 autour de $1 \%$ du PIB, tandis que le solde structurel primaire est excédentaire de plus de 2 points de PIB. La période 1989-1991 voit le retour des politiques budgétaires "actives" devant la nouvelle dégradation de l'activité économique, en particulier au Royaume-Uni. S'y ajoute l'impact budgétaire de la réunification allemande (de l'ordre de 0,8 point du PIB européen). À partir de 1991, c’est-à-dire du traité de Maastricht, si le solde public reste fortement déficitaire $(-4,4 \%$ du PIB en 1996), le solde structurel primaire se redresse fortement ( $+4 \%$ du PJB en 1996). Rien n'indique un laxisme excessif des politiques budgétaires.

Un niveau trop élevé des déficits publics aurait dû se traduire par l'apparition de déséquilibres importants sur le marché des biens. Ceux-ci n'ont pas été observés : au contraire, les années de forts déficits publics ont été des années de forte désinflation $(3,8 \%$ d'inflation pour l'UE en 1993 contre 8,5\% en 1983), de fort chômage, de marges importantes de capacités de production inemployées et de balances extérieures équilibrées. Aussi les politiques budgétaires ont-elles été prises dans un étau : soit laisser croître les detres publiques, soit peser lourdement sur un niveau de demande déjà trop faible. Le seul indice de déséquilibre est celui du niveau des taux d'intérêts, qui sont passés de 3 points en dessous du taux de croissance avant 1980 à 2 points au-dessus depuis. En 1993, le taux d'intérêt réel de court terme était de l'ordre de $5 \%$ en Europe (tandis qu'il était proche de 0 aux États-Unis et de $1,5 \%$ au 
Japon) alors même que le taux de croissance était négatif (de 0,4\%). Ces taux d'intérêt font peser une lourde charge sur les politiques budgétaires. Dans les années 1977-1979, la stabilisation du ratio dette/PIB était compatible avec un déficit primaire de l'ordre de 0,5 point de PIB; dans les années 1994-1995, c'est un excédent primaire de l'ordre de 1,6 point de PIB qui était nécessaire. Comment expliquer la coexistence en Europe de forts taux d'intérêt, de forts déficits publics er de niveaux d'activité relativement faibles comparés au niveau potentiel? Deux thèses s'opposent : le schéma keynésien et le schéma classique.

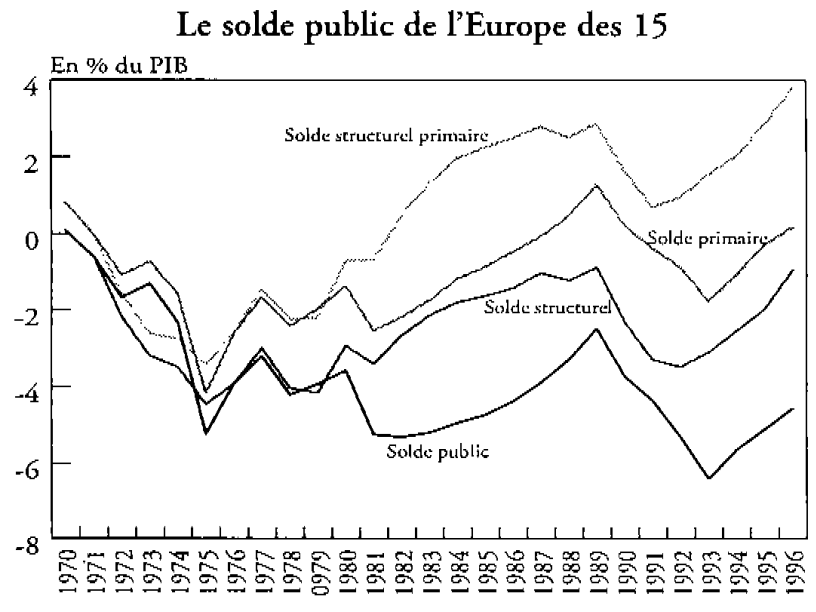

Dette publique nette de l'Europe des 15

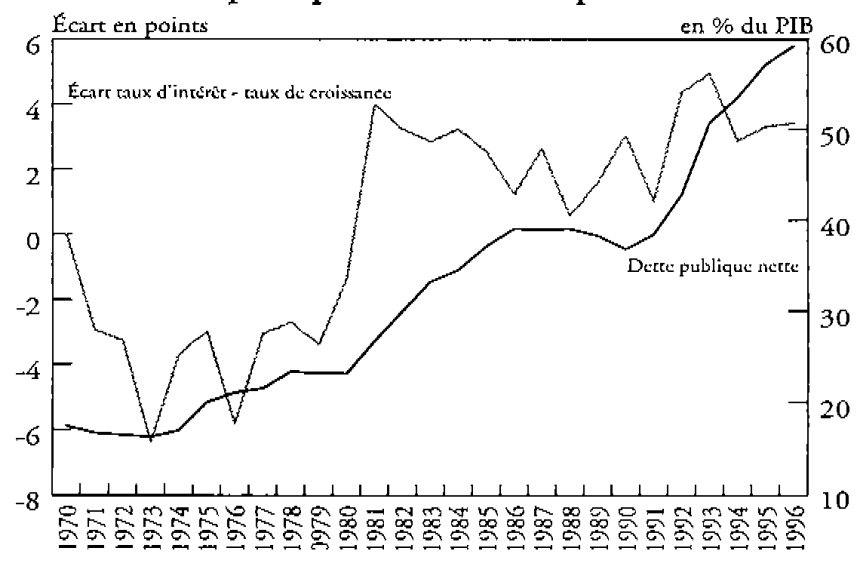




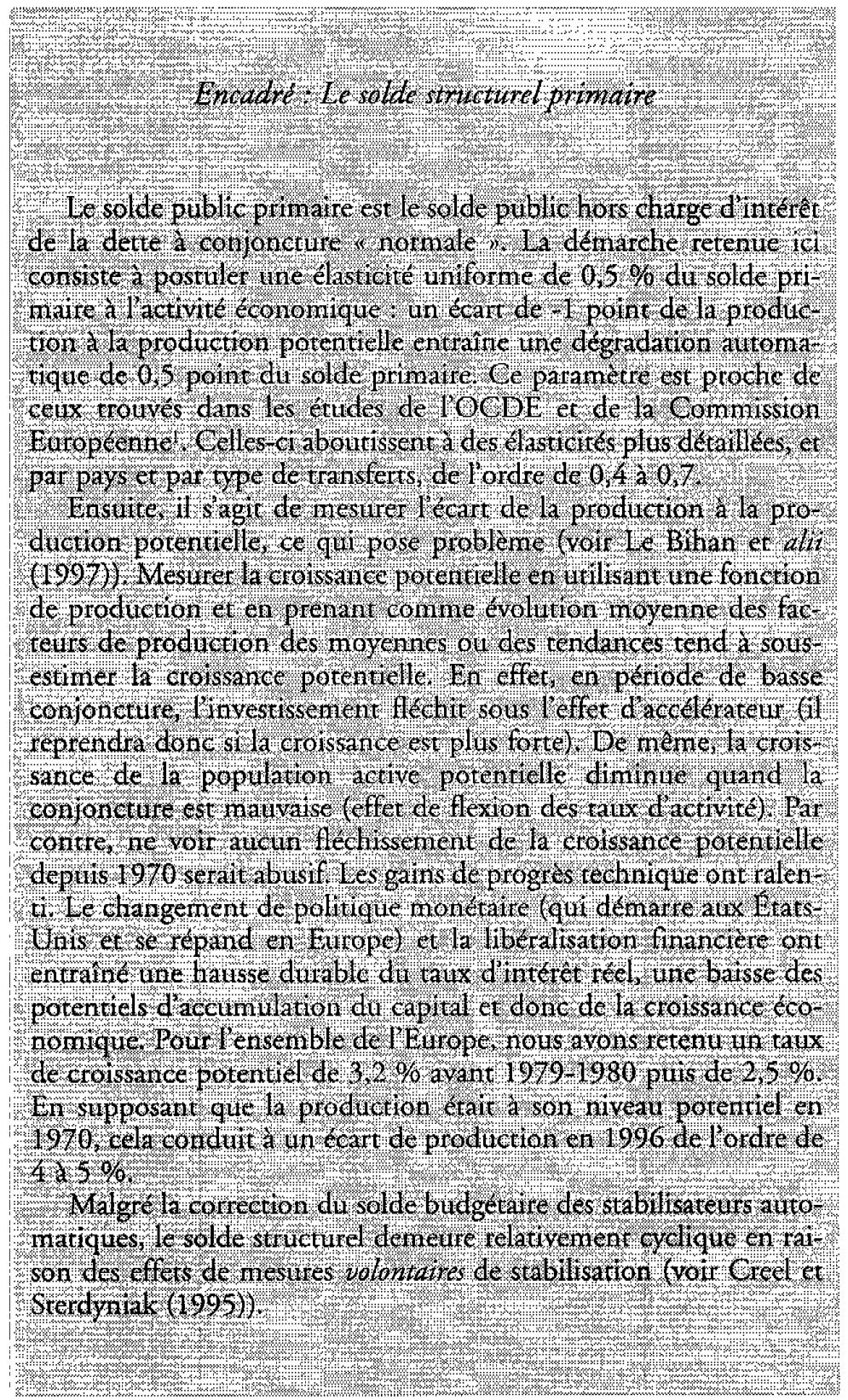


DU BON USAGE DES POLITIQUES MONETAIRE ET BUDGETAIRE EN EUROPE

\section{POLITIQUE BUDGETAIRE ET MONETAIRE EN ECONOMIE FERMEE}

Le traité de Maastricht amène à se poser la question du partage des rôles entre politique monétaire et politique budgétaire.

\section{Deux remarques introductives}

a) Nous supposerons ici que la politique monétaire consiste à fixer à chaque période le taux d'intérêt. Cette hyporhèse est conforme aux pratiques effecrives des banques centrales. Le contrôle de l'offre de monnaie imposerait que la réaction de la Banque centrale soit dictée par les paramètres de la demande de monnaie et non par ceux de sa fonction de perte elle-même; elle poserait problème en cas de chocs affectant spécifiquement la demande de monnaie. Elle impliquerait que la Banque centrale ait un objectif en terme de niveau des prix et non un objectif en terme de taux d'inflation, ce qui est peu réaliste. Malheureusement la quasi-totalité des modelés théoriques font l'hypothèse que la politique monétaire contrôle directement un instrument nominal (la masse monétaire) tandis que la politique budgétaire contrôle des instruments réels (les dépenses publiques en volume et les taux d'imposition). Aussi, ces modèles arrivent-ils à la conclusion qu'à long terme, le taux d'inflation n'est déterminé que par la politique monétaire. Mais, il s'agit là d'un pur artefact : ce résultar disparaît si la politique monétaire est décrite de façon plus réaliste, comme fixant à chaque période le taux d'intérêt, en fonction d'une certaine fonction de perte des autorirés monétaires. Dans ce cas, la politique budgétaire et la politique monétaire ont des rôles symétriques dans la détermination à chaque période de l'inflation et du niveau de production.

b) De nombreux résultats dépendent de l'influence comparée de la politique budgétaire et monétaire sur l'inflation. Pour poser simplement le problème, résumons le fonctionnement de l'économie en deux équations :

- la demande de biens : $y=\mathrm{kg}-\sigma_{\mathrm{r}}$

- les prix : $\mathrm{p}=\mathrm{ay}+\theta \mathrm{r}$

$\mathrm{g}$ étant un indicateur de la politique budgétaire ${ }^{2}$ et $\mathrm{r}$ un indicateur de la politique monétaire. Le point crucial est celui de l'impact spécifique des politiques budgétaire et monétaire, c'est-à-dire de la valeur de $\theta$. Si elle est négative, il est souhaitable d'affecter la politique budgétaire au soutien de l'activiré et la politique monétaire à la lurte contre l'inflation ${ }^{3}$. Une politique monétaire restrictive et une politique budgétaire expansionniste permetrent à la fois de réduire l'inflation et de soutenir l'activité. Si elle est positive, c'est l'affectation inverse qui est optimale ${ }^{4}$. 
Selon la théorie de "l'entonnoir commun ", les politiques budgétaire et monétaire ne jouent sur l'inflation que par le canal commun de leur influence sur la demande; aussi, à effet donné sur l'activité, leur impact sur l'inflation est le même ( $\theta$ est nul). Dans ce cas, les deux instruments sont redondants; les uriliser tous les deux en même temps de façon contradictoire ne peut aboutir qu'à un équilibre non satisfaisant avec des variations excessives des instruments.

En économie, fermée, une politique monétaire restrictive a un désavantage relatif pour lutrer contre l'inflation ( $\theta$ est positif). En effet, la hausse des taux d'intérêt frappe particulièrement les entreprises, qui sont débitrices nettes. Si celles-ci fixent leurs prix sur la base d'un coût de développement incluant le coût du capital ou si elles répercutent leurs charges financières dans leurs prix, ceux-ci sont une fonction croissante du taux d'intérêt. De même, si les entreprises réduisent leurs investissements en situation de taux d'intérêt élevés, une politique monétaire restrictive se traduit par une moindre accumulation de capital, donc par de plus fortes tensions sur le marché des biens. Aussi, est-il préférable d'utiliser la politique budgétaire pour lutter contre l'inflation et la politique monétaire pour soutenir l'investissement des entreprises.

Par contre, certains pensent qu'une politique monétaire expansionniste aurait un impact spécifique sur l'inflation ( $\theta$ serait négatif), donc qu'il faudrait affecter spécifiquement la politique monétaire au contrôle de l'inflation. Quels seraient les canaux de cet impact spécifique? On trouve deux arguments dans la littérature (voir Mundell, (1993)). Le premier repose sur les anticipations des agents économiques : ceux-ci considéreraient qu'une politique monétaire expansionniste entraînerait obligatoirement de l'inflation. Cette anticipation provoquerait une hausse immédiate des salaires, des prix et des taux d'intérêt de long terme. Mais, cet argument, totalement circulaire, est contradictoire avec la théorie des anticipations rationnelles qui enseigne que l'on ne peut baser un mécanisme de transmission sur de pures anticipations, sans lien avec un mécanisme réel. On voit mal pourquoi les agents considéreraient qu'une politique de soutien de la demande par la baisse des taux d'intérêt serait plus inflationniste qu'une politique de soutien par le déficit public alors que la première réduit les charges des entreprises, favorise le développement des capacités de production, est plus facilement réversible, tandis que la seconde augmente le déficit et la dette publics.

En second lieu, en économie ouverte, en change flexible, une politique monétaire restrictive permet certes de lutter contre l'inflation à moindre coût en activité, à court terme, puisqu'elle provoque une appréciation de la parité, donc une baisse des prix des produits importés, qui se répercute dans les prix à la consommation. Mais, cette stra- 
tégie est fragile. En effet, elle provoque un choc inflationniste chez les partenaires du pays qui l'entreprend. Au niveau mondial, l'avantage relatif de la politique monétaire disparât puisqu'il ne réside pas dans une capacité spécifique à réduire l'inflation, mais seulement dans une capaciré spécifique à l'exporter chez ses partenaires. Si tous les pays pratiquent la même politique, l'effet souhaité de surévaluation ne se produit pas et le gain spécifique espéré en utilisant la polirique monétaire se révèle illusoire. Si un pays est le seul à se lancer dans une telle stratégie, la surévaluation de son taux de change provoque des pertes de compétitivité, donc un déficit extérieur qui n'est guère soutenable. Tôt ou tard, il doit retrouver une compétitivité satisfaisante, donc accepter que, soit par retour de son taux de change nominal à sa valeur initiale, soit par une politique restrictive qui fasse pression sur les salaires, ses prix internes augmentent moins vite que les prix de ses concurrents. Durant cette phase de reconstitution de sa compétitivité, les gains de désinflation rapide obtenus pendant la phase initiale sont payés par un impacr inflationniste des prix à l'importation. Au total, la politique monétaire restrictive ne permet que d'accélérer la désinflation et non d'en limiter le coût en activité. Cette accélération est payée par une accumulation transitoire de déficits commerciaux. Aussi, l'utilisation de l'arme monétaire en change flexible pose-t-elle des questions délicates de coordination des politiques économiques à l'échelle mondiale.

Déficits autonomes, déficits de régulation : le schéma keynésien

À court terme, le gouvernement doit choisir un objectif de niveau de production. Celui-ci peut correspondre au taux de chômage naturel si le taux d'inflation est satisfaisant et si le niveau du taux de profit est suffisant pour que les entreprises puissent financer l'investissement nécessaire; il doit être temporairement inférieur à celui-ci s'il est nécessaire de faire pression sur les salaires, soit pour faire baisser l'inflation, soit pour augmenter le taux de profit jusqu'à un niveau adéquat.

En situation de demande insuffisante ex ante, la politique optimale consiste à faire baisser le taux d'intérêt jusqu'à ce que la demande soit suffisamment relancée; cette politique a l'avantage de ne pas augmenrer la derre publique, de favoriser l'accumulation du capital et de réduire le taux de profit nécessaire. Elle peut se révéler insuffisante, en particulier parce qu'il y a un plancher à la baisse des taux d'intérêt nominaux, (donc réels si on se refuse à pratiquer de l'inflation) : en France, par exemple, en 1997, le taux d'intérêt au jour le jour ne descend guère au-dessous de $3 \%$, ce qui aboutit à un taux de base des banques commerciales de l'ordre de $6 \%$ et à un taux réel du crédit de $4 \%$. Pour obtenir un niveau de demande satisfaisant, le gouvernement doit alors 
accepter un certain déficit budgétaire, que nous nommerons déficit de régulation. Un tel déficit nécessaire pour soutenir l'activité n'a évidemment aucun effet d'éviction des dépenses privées : il ne provoque pas de hausse du taux d'intérêt, puisque par définition le taux d'intérêt est à son plus bas niveau possible. Il ne pose pas a priori de problème de soutenabilité si les mesures de relance prises sont effectivement temporatires : si l'accumulation de dette publique amène ultérieurement les agents à augmenter leurs dépenses, l'État pourra réduire son déficit du montant nécessaire. De plus, une hausse permanente des dépenses publiques provoque en situation de chômage keynésien une hausse de la production à moyen terme telle que la hausse induite des recettes fiscales compense le déficit initial (Christ, (1968)). Les ménages ne doivent donc pas, s'ils sont rationnels, anticiper qu'une hausse des dépenses publiques donnera lieu à une hausse des impôts futurs. A contrario, en situation de chômage keynésien et de déficit de régulation, il ne peut pas exister de " contractions budgétaires expansionnistes ", contrairement à ce qu'ont voulu démontrer Giavazzi et Pagano (1990).

Un cas de figure différent apparaît lorsque la demande étant à un niveau satisfaisant, le gouvernement provoque un déficit public suite à des décisions d'augmenter les dépenses, sans hausse compensatrice des rentrées fiscales : c'est ce que nous nommerons un déficit autonome. Il en résulte un excès de demande sur le marché des biens, qui a des effets expansionnistes et inflationnistes. Ces derniers obligent soit à pratiquer une hausse des taux d'intérêt particulièrement nuisible, qui freine effectivement la demande mais par baisse de l'investissement et donc au détriment des possibilités de croissance, soit à accepter une hausse du taux d'inflation (mais celle-ci ne réduit guère la demande si elle n'est pas accompagnée d'une hausse des taux d'intérêt réels, car dans les économies modernes la plupart des actifs financiers sont rémunérés à des taux de marché). Ce type de déficit pose un problème de soutenabilité puisque l'accumulation de dettes publiques renforce de façon continue les tensions sur le marché des biens.

Il convient donc de pratiquer des déficits de régulation et d'éviter les déficits autonomes. La distinction ne va pas de soi concrètement, mais elle est utile à des fins descriptives et normarives. Le point essentiel est qu'elle ne peut être basée sur un critère mécanique, tel que l'importance du déficit relativement au PIB, mais qu'elle doit tenir compte de la rature des déséquilibres sur le marché des biens. Aussi le seul critère pertinent est-il qu'un déficit public est excessif lorsqu'il entraîne un excès de demande, c'est-à-dire quand il oblige à pratiquer des taux d'intérêt trop forts ou lorsqu'il provoque un niveau d'inflation et un déficit extérieur supérieurs aux niveaux souhaités. De même, une dette 
publique ne peut être jugée excessive que lorsqu'eile entraîne (par effet de richesse) une consommation trop forte des ménages et donc un excès de demande. Le fait est que la plupart des pays de la CE qui ont actuellement un fort niveau de dette publique (Belgique, Italie, Pays-Bas) ont en même temps un fort taux d'épargne des ménages. Ce n'est d'ailleurs qu'en raison de ce fort taux d'épargne qu'ils ont dû et pu accumuler cette dette : un fort déficit public était compatible chez eux avec un niveau satisfaisant de demande et n'entraînait pas de déséquilibre extérieur insoutenable.

Lévolution récente des politiques économiques en Europe n'en demeure pas moins paradoxale. Comment justifier la coexistence de taux d'intérêt élevés et de déficits primaires, qui pose des problèmes de soutenabilité des finances publiques? Dans une problématique keynésienne, une demande insuffisante nécessite à la fois une hausse du déficit public, mais aussi une baisse des taux d'intérêt réels qui en atténue l'impact sur l'évolution de la dette publique; c'est ce qui fut réalisé entre 1974 er 1977. Pourquoi ce mécanisme ne fonctionne-t-il plus aujourd'hui? Trois schémas prétendent expliquer ce paradoxe.

\section{Le paradigme néo-classique}

Le paradigme néo-classique fournit une explication simple et cohérente des évolutions constatées des déficits publics er des raux d'intérêt (voir notamment Phelps, (1994)). L'hypothèse de base est que le taux d'intérêt réel égalise l'offre et la demande de biens. Les salaires réels sont rigides (en raison de règles d'indexation, des exigences des travailleurs en place ou en raison de mécanismes de salaire d'efficience). L'offre de biens est une fonction décroissante du taux d'intérêt réel, dont la hausse amène les entreprises à augmenter leurs prix et à réduire l'accumulation de capital. La demande de biens dépend positivement des dépenses publiques, de la dette publique et négativement du taux d'intérêt réel. Le taux d'intérêt réel reflète donc les tensions sur le marché des biens : un niveau élevé de celui-ci correspond donc à un excès de demande (autrement dit : une insuffisance d'épargne). Une hausse de demande (due à une baisse du taux d'épargne privé ou à une hausse du déficit public) provoque une hausse du taux d'intérêt réel er une baisse de la production. La politique monéraire ne joue aucun rôle dans la détermination du taux d'intérêt réel. Enfin, la stabilité de l'économie nécessite que l'État pratique une politique restrictive en cas de forr taux d'intérêr, de forte dette publique, et de baisse de la production (qui ne peut résulter dans ce paradigme que de chocs d'offre). Par contre, l'économie est instable si le gouvernement pratique une politique keynésienne, en relançant les dépenses publiques lorsque la production diminue. 
Selon ce modèle, les forts niveaux de déficits publics (ou la baisse de l'épargne privée) sont responsables du fort niveau des taux d'intérêt, eux-mêmes responsables de la baisse de la production. En sens inverse, pour sortir de la crise, il faut réduire les déficits publics (ou augmenter l'épargne). Ceci peut être pénible (en obligeant à réduire la consommation ou les dépenses publiques), mais a des effets macroéconomiques favorables : les taux d'intérêt baissent, l'investissement prend le relais des dépenses publiques et la production augmente. Au total, la réduction des déficits publics et la hausse du taux d'épargne devraient être une priorité en Europe à l'heure actuelle : les critères de Maastricht sont donc validés par l'analyse économique.

Ce modèle a donc des conséquences fortes pour la conduite de la politique économique. Toutefois, il présente plusieurs défauts dirimants. En premier lieu, il n'explique pas pourquoi la totalité des pays d'Europe ont en même temps accru leur déficit public, donc se seraient engagés dans des politiques absurdes du point de vue macroéconomique. Ensuite, il manque une étape dans le raisonnement : il faudrait expliquer le mécanisme selon lequel le taux d'intérêt réel équilibre le marché des biens, compte tenu du fair que la Banque centrale fixe le taux d'intérêt nominal de court terme. Ce mécanisme ne peut jouer qu'en situation de pleine utilisation des capacités de production : ce n'est pas globalement le cas en Europe depuis 1981 Enfin, il ne correspond en rien aux évolutions constatées : le taux d'intérêt réel devrait être élevé dans les périodes où la demande est forte par rapport à l'offre, donc dans les périodes de forte inflation et de forts taux d'utilisation des capacités de production. Sans même évoquer la situation du marché du travail, force est de constater que l'Europe a vécu (sauf durant un court épisode en 1988-1989) avec un niveau important de marges de capacités inemployées. Comment expliquer sinon par une rupture de comportement de la politique monétaire, que le taux d'intérêt réel était de l'ordre de $-2 \%$ entre 1969 et 1972 , années de fortes tensions sur les capacités de production, et de 3,5\% entre 1981 et 1987, années de basse utilisation des capacités de production? La forte hausse des taux d'intérêt réels en 1980-1981 ne correspond pas à un fort choc de demande et à une forte utilisation des capacités de production. De même, le fort ralentissement de la demande dans les années 1991 à 93 ne s'est pas accompagné d'un essor de la production : les taux d'intérêt réels ont certes légèrement baissé (de 7 à $5 \%$ pour le taux de court terme), mais cela n'a pas été suffisant pour empêcher le ralentissement économique. Aussi, les gouvernements ont-ils soutenu l'activité par la politique budgétaire ne voulant pas, par une politique restrictive, ajouter un facteur supplémentaire de dépression. Rien ne leur permettait de 
croire à la fiabilité de la stratégie inverse : réduire le déficit public pour provoquer une baisse suffisante des taux d'intérêt qui soutiendrait l'activité. Les gouvernements se sont retrouvés keynésiens, en acte, si ce n'est en paroles : il y a une contradiction entre avoir accepté une forte hausse des déficits publics en 1991-1993 et s'être engagés à les réduire dans le traité de Maastricht puisqu'on reconnaîr, en fair, qu'un forr déficit public peut être parfois nécessaire en même temps qu'on considère possible de prendre des engagements non circonstanciés sur son niveau futur. Cetre schizophrénie ne permer guère d'envoyer des signaux clairs aux marchés. Ainsi, une proposition telle que "Les déficits publics sont responsables des forts niveaux des taux d'intérêt ", qui équivaut à "le taux d'intérêt équilibre l'offre et la demande de biens ", n'a de sens qu'en situation de plein-emploi des capacités de production. Elle ne rend pas compte de l'évolution économique récente et ne peur donc servir de guide à la politique économique.

\section{La hausse de la dette publique comme contrepartie au désendettement des entreprises}

La modification du niveau des taux d'intérêt réels entre les années soixante-dix et les années quatre-vingt/quatre-vingt-dix ne correspond pas à un changement de niveau structurel des tensions sur le marché des biens (voir Creel et Sterdyniak (1995)). Aussi, sa cause doir-elle être recherchée au niveau du fonctionnement des marchés financiers. La hausse des taux d'intérêt réels est due à l'utilisation de la politique monétaire pour lutter contre l'inflation, à la désinflation, à la dérégulation financière er à la libéralisation des marchés de capitaux. L'économie européenne connaissait une situation marquée par une inflation importante et un comportement monopolistique du système financier : celuici rémunérait peu une partie imposante des déposants et répercutait ce gain sur les emprunteurs. Aussi, les taux d'intérêt nominaux étaient bas et les taux d'intérêt réels nuls ou négatifs. Cette situation était encouragée par les gouvernements qui y voyaient un moyen de soutenir l'investissement. L'économie européenne est passée depuis à un autre régime : l'utilisation de la politique monétaire pour lutter contre l'inflation (aux États-Unis d'abord) s'est propagée en Europe avec la déréglementation financière et la libéralisation des marchés de capitaux. Ceux-ci ont brisé les monopoles bancaires; la concurrence des intermédiaires financiers a fait disparâtre les ressources à bon marché; la concurrence entre pays a obligé chacun à s'aligner sur le taux d'intérêt réel le plus élevé offert. La désinflation a fait disparaître la prime inflationniste. Comme, en même temps, le ralentissement des gains de productivité s'est accentué en Europe, l'écart s'est creusé entre le taux d'intérêt et le taux de croissance. 
L'évolution des taux d'intérêt est de moins en moins contrôlée par les gouvernements; de plus en plus dictée par les marchés avec l'irrationalité et la myopie qui les caractérisent (voir Lordon (1994)). Or, dans une économie monétaire, la fixation du taux d'intérêt n'est pas un phénomène de marché. C'est le fondement même de la théorie macroéconomique qu'il n'existe pas et ne peut exister de mécanisme qui assurerait ex ante l'égalité entre les actifs financiers émis et ceux que les agents veulent détenir, c'est-à-dire entre l'épargne et l'investissement. Le taux d'intérêt ne peut équilibrer l'épargne et l'investissement, car c'est l'investissement qui crée l'épargne que ce soir par variation du niveau de production ou par variation du niveau des prix. L'équilibre peut se réaliser avec un taux d'intérêt élevé et un bas niveau d'activité; comme àvec un taux d'intérêt bas et un niveau d'activité plus élevé. Dans les deux cas, il n'y a pas de forces automatiques sur le marché des capitaux qui tend à faire évoluer le taux d'intérêt vers un niveau correspondant à un équilibre satisfaisant du marché des biens. C'est ce que ne voient pas Aglietta, Coudert et Mojon (1993), qui écrivent : «Actuellement, dans un système libéralisé et intégré, les variations des taux d'intérêt permettent d'ajuster l'équilibre épargne-investissemént, sans biais inflationnisthe durable ", sans s'interroger sur le biais restrictif du niveau actuel des taux d'intérêt en Europe. Certes, les anticiparions des marchés déterminent les taux d'intérêt de long terme, mais ceux-ci ne peuvent l'être que sur la base des taux d'intérêt de court terme futurs anticipés. Or, les marchés n'ont aucune prescience de l'équilibre macroéconomique de demain et ont intérêt au maintien de tauxx d'intérêt élevés. L'économie peut donc être piégée dans un équilibre de sous-emploi des capacités de production, de faible inflation, de fort taux d'intérêt réel. Dans ce contexte, il est vain d'espérer qu'une réduction des déficits publics entraînera une baisse des taux d'intérêt par pur effet de marché, puisque dette réduction conduira à une chute de la production, donc une baisse de l'épargne.

Quelles sont les conséquences macroéconomiques d'une hausse exogène de l'écart entre taux d'intérêt et taux de croissance dans un modèle avec comportements patrimoniaux? La profitabilité des entreprises est dégradée, celles-ci veulent donc réduire leur ratio d'endettement, elles diminuent fortement leurs investissements. La hausse du taux d'intérêt permet aux ménages de consommer plus, tout en ayant un patrimoine plus élevé. Supposons tout d'abord que le gouvernement ne dispose que d'un seul instrument de politique économique, qui ne lui permette que de gérer le niveau de demande (les dépenses publiques ou le taux d'imposition portant sur les ménages). Il le fait varier de façon à maintenir la demande au niveau des capacités de production. À la suite 
de la hausse du taux d'intérêt, les ménages augmentent leur consommation, les entreprises réduisent leur investissement. Pour stabiliser le niveau de production, le gouvernement doit pratiquer une politique expansionniste. Son déficit augmente aussi en raison de l'augmentation des charges d'intérêt. Cette politique permet de stabiliser à court terme le niveau de production, mais l'équilibre est obtenu avec une consommation plus forte er un investissement plus faible que dans le sentier de croissance initial. À moyen terme, ce bas niveau d'investissement entraîne un ralentissement de la croissance des capacités de production, donc de la production. La politique budgétaire doit devenir restrictive pour réduire le niveau de la demande. Toutefois, la dette publique reste importante. La croissance étant insuffisanre, le taux de chômage croît sans cesse. Au départ de cette évolution, le taux d'épargne des ménages est bas, la dette et le déficit publics importants, l'observateur pourrait donc conclure à un déficit d'épargne, mais il n'en est rien : le problème réside dans un déficit d'investissement, dû au taux d'intérêt trop élevé.

Pour stabiliser parfaitement l'économie, les aurorités doivent disposer de deux instruments, l'un pour contrôler la demande, l'aucre (le taux d'intérêt ou sinon les impôts sur les entreprises) pour contrôler l'offre. Imaginons qu’à la suite de la hausse des taux d'intérêt, l'État réduise les impôts portant sur les entreprises pour que celles-ci maintiennent leurs investissements. Ceci revient à ce qu'il prenne en chatge la hausse des frais financiers portant sur les entreprises, ainsi que le supplément de profit nécessaire résultant du désir de celles-ci de se désendetter. Par contre, il augmente les impôts portant sur les ménages pour éviter que ceux-ci n'augmentent leur consommation Ceci se fait au détriment des travailleurs dont les impôts augmentent, mais le plein-emploi est maintenu. La hausse des taux profite aux rentiers. Quant aux entreprises, la baisse des impôts compense la hausse des taux d'intérêt. Les impôts sur les ménages augmentent donc du montanc d'intérêt supplémentaire que ceux-ci reçoivent. Cette politique permer le maintien en permanence du plein-emploi à moyen-terme. Par contre, en croissance équilibrée, l'Êtat doit avoir un déficit plus fort et une dette supplémentaire, qui correspond juste au désendetrement désiré par les entreprises. Cette hausse de la dette publique est, en quelque sorte, désirée par le secteur privé pris globalement. Le déséquilibre des finances publiques permet donc de maintenir l'équilibre macroéconomique.

Ce modèle patrimonial permet d'illustrer quelques points importants : la hausse du taux d'intérêt par rapport au taux de croissance a aussi des conséquences importantes pour l'équilibre des agents privés : on ne peut en discuter l'impact sur les finances publiques sans tenir compte du comportement des ménages et des entreprises. Elle nécessi- 
te une hausse de la dette publique, qui correspond à la baisse de l'endettement désiré des entreprises. Une politique budgétaire active doit compenser la perte de contrôle de la polirique monétaire.

\section{Lindépendance de la Banque centrale}

L'indépendance de la Banque centrale peut expliquer des orientatilons différentes des politiques monétaires et budgétaires, dans une situation keynésienne comme dans une situation classique (voir Capoën, Sterdyniak et Villa (1994)). La politique budgétaire et la politìque monétaire ont des rôles symétriques dans la détermination à chaque période de l'inflation et du niveau de production. À taux d'inflation stable, il existe un lien étroit entre la politique budgétaire (fixation des dépenses publiques) et la politique monétaire (fixation du taux d'intérêt réel). Il est impossible d'envisager une configuration où la politique monétaire et la politique budgétaire seraient indépendantes à moyen terme. Si la demande privée augmente, il faut choisir entre augmenter les impôts ou augmenter le taux d'intérêt.

Si la Banque centrale n'est pas indépendante, les autorités gouvernementales réagissent à un choc inflationniste ou à une hausse de la demande en augmentant le taux d'intérêt réel et en réduisant les dépenses publiques. Leur réaction dépend de leur répugnance relative pour la hausse des taux d'intérêt, la baisse des dépenses publiques et la hausse des impôts.

Que se passe-t-il si les autorités monétaire et budgétaire sont indépendantes et ont des fonctions de perte différentes? Dans le cas où l'utilissation des instruments de politique économique n'a pas de coût, le problème n'a pas de solution si les autorités agissent de façon indépendante (en réalisant un équilibre de Nash). Si le niveau de production (et le niveau d'inflation) se trouvent en dessous du niveau désiré par les autorités monétaires et au-dessus de ceux désirés par les autorités budgétaires, les autorités monétaires vont augmenter indéfiniment le taux d'intérêt tandis que les autorités budgétaires vont augmenter indéfiniment le déficit public. Il est impossible de confier la responsabilité de la politique conjoncturelle à deux autorités indépendantes. On ne peut ếchapper à ce risque de dérive qu'en décidant qu'une seule des autorités a la responsabilité de la politique conjoncturelle.

Ce conflit est atténué si les fonctions de perte des deux autorités domportent un coût d'utilisation des instruments. Dans l'équilibre de Nash où chaque autorité réagit en prenant comme donnée la politique de l'autre, le niveau de production d'équilibre apparaît comme une pondération des désirs des deux autorités; mais cette pondération ne s'effectue qu'à partir des répugnances que chacune d'elle a à utiliser son 
propre instrument de politique économique. Par exemple, si la Banque centrale ne ressent aucun coût à faire varier le taux d'intérêt et si le gouvernement répugne à creuser le déficit budgétaire, c'est la Banque centrale qui impose le niveau de production. C'est l'inverse si la Banque centrale répugne à augmenter le taux d'intérêt tandis que le gouvernement n'hésite pas à recourir au déficit public. Aussi, le niveau de production atteint ne présente-t-il aucun caractère d'optimalité. De plus, le niveau des instruments dépend à l'équilibre des écarts entre les fonctions de perte des autorités : le taux d'intérêt et le niveau du déficit public augmentent si le gouvernement souhaite lutter davantage contre le chômage tandis que la Banque centrale souhaite limiter davantage l'inflation. Aussi, cette configuration institutionnelle aboutit-elle à des variarions excessives des instruments de politique économique : le taux d'intérêt augmente à la fois pour freiner l'activité et pour compenser la hausse du déficit public, qui elle-même vise à soutenir l'activité et à lutter contre la hausse du taux d'intérêt. Les autorités auraient intérêt à négocier et à échanger une baisse du taux d'intérêt contre une baisse du déficit public; mais aucune des deux n'a intérêt à s'engager unilatéralement dans un tel processus.

Enfin, l'affectation spécifique de la politique monétaire à la lutte contre l'inflation qui est implicitement obrenu quand la Banque centrale est indépendante ne va pas de soi. Dans une situation inflationniste, elle induit une hausse des taux d'intérêt réels, qui d'une part provoque une hausse des charges d'intérêt des entreprises, donc renforce l'inflation par les coûts, d'autre part tend plutôt à augmenter la consommation des ménages (qui reçoivent plus de revenus d'intérêt) et à réduire l'accumulation du capital, ce qui peut à terme renforcer les tensions sur le marché des biens. Lutter contre l'inflation par la politique budgétaire serait done préférable.

\section{POLITIQUE BUDGÉTAIRE ET POLITIQUE MONÉTAIRE EN ECONOMIE OUVERTE}

En économie fermée, le gouvernement peut choisir la combinaison de politiques monétaire et budgétaire permettant d'obtenir et de maintenir le niveau désiré de demande. Ce degré de liberté peut lui permettre d'affecter la politique monétaire au maintien de la profitabilité des entreprises et la politique budgétaire au maintien de la demande. En économie ouverte, les deux instruments ont des impacts différents sur le taux de change, et donc sur les pays partenaires, sont soumis à des contraintes extérieures différentes. Nous nous intéresserons d'abord à 
l'organisation de la politique économique en change fixe (en prenant l'exemple du SME); puis nous comparerons ce cas avec celui des changes flexibles; enfin, nous nous interrogerons sur le fonctionnement de l'UEM.

\section{Le modèle}

Le monde se compose de deux pays identiques. L'équilibre sur le marché des biens s'écrit pour le premier pays (non étoilé) :

(1) $y=g+d+c y-\sigma r+k w+n\left(y^{*}-y\right)+n \delta\left(p^{*}+s-p\right)$

et pour le second pays (éroilé) :

(2) $y^{*}=g^{*}+d^{*}+c y^{*}-\sigma r^{*}+k w^{*}-n\left(y^{*}-y\right)-n \delta\left(p^{*}+s-p\right)$

où y représente la production, $g$ les dépenses publiques, $r$ le taux d'intérêt, $\mathrm{p}$ le niveau des prix de production, $w$ la richesse des agents privés. Les variables étoilées correspondent au second pays. Tous les paramètres sont positifs. Nous supposons que l'économie souffre d'une contrainte de débouchés et que les prix sont rigides à court terme, l'offre de biens s'ajuste donc à la demande de biens. La demande privée est égale à la somme de la consommation $(\mathrm{cy}+\mathrm{kw})$, de l'investissement $\left(d-\sigma_{r}\right)$ et des exportations nettes de chaque pays. Celles-ci dépendent de l'écart de conjoncture et de la compétitivité relative. La fonction de consommation prend en compte un effer de richesse.

La richesse nette des agents privés de chaque pays comprend la dette publique et les actifs détenus sur l'étranger.

(3) $w=f+b$

(4) $w^{*}=-f+b^{*}$

avec $b$ le niveau de dette publique et $f$ la dette du deuxième pays relativement au premier. Les équations d'accumulation d'actifs s'écrivent :

(5) $\mathrm{b}=\mathrm{g}-\mathrm{t}$

(6) $f=n\left(y^{*}-y\right)+n \delta\left(p^{*}+s-p\right)$

A court terme, les prix et les stocks d'actifs sont fixes. Les deux pays souhaitent maintenir la demande $(y)$ à un niveau égal à 0 , correspondant au taux de chômage naturel. Quelles sont les politiques économiques nécessaires à court puis à long terme?

\section{La logique des changes fixes}

Un régime de change fixe est obligatoirement un régime dissyméthique où un pays dominant fixe librement sa politique monétaire tandis que les pays dominés doivent consacrer leur politique monétaire au maintien de la fixité du taux de change. Le taux d'intérêt de la zone, fixé par le pays dominant, ne correspond pas nécessairement aux besoins et 
aux désirs des pays dominés. Le SME n'a pas échappé à cette caractéristique, malgré les mécanismes mis en place au moment de sa création (l'écu et l'indicateur de divergence). Un système symérrique aurait nécessité une négociation permanente sur la fixation des taux d'intérêt, négociation qui aurait été contradictoire avec le souhait de la Bundesbank de conserver son indépendance et sa pleine capacité d'influencer le comportement des acteurs de la vie économique allemande et de préserver la valeur interne du DM. Nous écrirons donc que le pays dominant fixe librement son taux d'intérêt tandis que le pays dominé est contraint de fixer le sien à :

(7) $r^{*}=r+\theta f$

L'accumulation d'une dette extérieure fait peser sur le pays endetté une prime de risque proportionnelle à cette derre. Nous considérerons deux types de chocs permanents : le pays dominant subit un choc de demande privée, $d$, positif, qu'il ne peut ou ne veut compenser par la politique budgétaire (choc $A$ ). Le pays dominé subit un choc négatif de demande privée, $\mathrm{d}^{*}$ (choc $\mathrm{B}$ ). Le pays dominé a le choix a priori entre quatre stratégies :

- Maintien du plein-emploi sans changement de parité : dans la cas du choc $\mathrm{A}$, le pays dominant fixe le taux d'intérêt à la valeur $\mathrm{r}=\frac{\mathrm{d}}{\sigma}$; le pays dominé pratique alors une hausse des dépenses publiques telle que $\mathrm{g}^{*}=\mathrm{d}$, pour compenser l'effet dépressif de la hausse du taux d'intérêt. Les deux pays restent au plein-emploi avec un fort taux d'intérêt; le déficit public du pays dominé est égal à l'excès de demande du pays dominant. Le pays dominant peut utiliser sa politique monétaire pour réguler son activité; le pays dominé doit utiliser sa politique budgétaire. Le pays dominé a été contraint de singer la situation du pays dominant. Dans le cas du choc B, le pays dominant ne modifie pas son taux d'intérêt; le pays dominé est contraint de faire une politique budgétaire expansionniste de stabilisation, qui compense la baisse de la demande privée. Il ne peut utiliser celui-ci pour faire baisser son taux d'intérêt. Le taux d'épargne du pays dominé n'influence pas le taux d'intérêt mondial. De même, il est impossible pour le pays dominé de réduire sa dette publique en maintenant le plein-emploi.

- Maintien de la parité et du solde public : Dans le cas du choc A, si le pays dominé se refuse à augmenter son déficit public, la hausse du taux d'intérêt est légèrement plus faible, $r=\frac{d(1-c+n)}{\sigma(1 c+2 n)}$, mais la chute de la production est importante dans le pays dominé. Elle est de $y^{*}=\frac{-d}{1-c+2 n}$. 
Le choc a priori expansionniste se transforme en choc récessif pour le pays dominé tandis que le pays dominant maintient le plein-emploi. Le pays dominant connaît alors un certain déficit extérieur; il accumule de lia dette extérieure, ce qui fait progressivement baisser le niveau de sa demande. À long terme, les deux pays retournent au plein-emploi quand la perte d'actif accumulée par le pays dominant est de $\mathrm{f}=\frac{-\mathrm{d}}{2 \mathrm{k}+\theta \dot{\sigma}}$. La hausse du taux d'intérêt est de $r=\frac{k+\theta \sigma}{2 k+\theta \sigma} \mathrm{d}$ dans le pays dominant; la baisse du taux d'intérêt est de $\mathrm{r}^{*}=\frac{-\theta \sigma}{2 \mathrm{k}+\theta \sigma} \mathrm{d}$ dans le pays dominé. Toutefois, le retour à l'équilibre prend du temps et demande une phase transitoire de chômage dans le pays dominé. Dans le cas du choc B, la dynamique est similaire. Au départ, la chute de la production est de $y^{*}=\frac{-d}{1-c+2 n}$ tandis que la baisse du taux d'intérêt mondial est de $r=\frac{d(1-c+n)}{\sigma(1-c+2 n)}$. terme, la production du pays dominé se redresse grâce à l'accumulation d'actifs extérieurs jusqu'à ce que $\mathrm{f}=\frac{-\mathrm{d}^{*}}{2 \mathrm{k}+\theta \sigma}$. À ce moment, la baisse du taux d'intérêt est de $\mathrm{r}=\frac{-\mathrm{k}}{\sigma(2 \mathrm{k}+\theta \sigma)} \mathrm{d}^{*}$ dans le pays dominant et de $\mathrm{r}^{*}=\frac{\mathrm{k}+\theta \sigma}{(2 \mathrm{k}+\theta \sigma)} \mathrm{d}^{*}$

dans le pays dominé. De façon similaire, le pays dominé peut donc téduire le poids de sa dette publique de $-\mathrm{b}^{*}$ en acceptant une baisse transitoire de sa productionet en accumulant un montant $\mathrm{d}^{\prime}$ avoir extérieur de $f^{*}=\frac{k b^{*}}{2 k+\theta \sigma}$; ceci nécessitant une perte cumulée de production de $\mathrm{y}^{*}=\frac{-\mathrm{kb}^{*}}{2 \mathrm{k}+\theta \sigma}$.

Le coût de cette réduction est plus fort que si le pays était un pays domihant ou un pays en économie fermée puisque le pays est contraint de subir une baisse de production durant la période transitoire au lieu de pouvoir compenser la baisse ex-ante de la demande par une baisse de son taux d'intérêt.

- Maintien du plein-emploi avec changement de parité : Une autre stratégie consiste en une modification de la parité au moment du choc. Dans le cas du choc A, par exemple, le pays dominé pourrait ne pas modifier sa politique budgétaire; par contre, il dévaluerait son taux de change de $s=0,5 \frac{\mathrm{d}}{\mathrm{n} \delta}$ La hausse du taux d'intérêt serair alors de $\mathrm{r}=0,5 \frac{\mathrm{d}}{\sigma}$ 
puisque le marché des biens du pays dominant s'équilibre en partie grâce à un déficit commercial vis-à-vis du pays dominé égal à la moitié du choc initial. Pour le pays dominé, l'excédent commercial compense la hausse du taux d'intérêt. Cette stratégie limite donc la hausse du taux d'intérêt et permet le maintien du plein-emploi dans les deux pays. Le point délicat est que, par la suite, l'accumulation des déficits courants du premier pays par rapport au deuxième amène une baisse de la demande dans le premier pays, une hausse dans le deuxième de sorte que la nouvelle parité n'est pas tenable. Le taux d'intérêt va augmenter dans le premier pays pour compenser la baisse de la demande et diminue dans le second par rapport à sa valeur de court terme. La monnaie du premier pays doit être dévaluée progressivement. La fixité des changes est donc incompatible avec les ajustements nécessaires au cours de la période transitoire.

- Maintien du plein-emploi avec modification des prix relarifs : envisageons enfin la possibilité d'une modification des prix relatifs. Celle-ci pourrait être obtenue par un ajustement des salaires. Toutefois, on voit mal comment celui-ci pourrait être acquis : si les salaires évoluent selon une courbe de Phillips augmentée, il faut, par exemple, une période transitoire de chômage pour faire baisser le niveau des salaires réels. Or, l'objectif est au contraire de maintenir le plein-emploi. Il faut donc envisager une politique budgétaire active : par exemple, une baisse des cotisations employeurs accompagnée d'une hausse des impôts portant sur les ménages. Dans le cas du choc $\mathrm{A}$, le pays dominé doit obtenir une baisse des prix de $\mathrm{p}=-0,5 \frac{\mathrm{d}}{\mathrm{n} \delta}$. L'effet macroéconomique est le même que pour une dévaluation. La hausse du taux d'intérêt est de $\mathrm{r}=0,5 \frac{\mathrm{g}}{\sigma}$. Cette politique, si elle est réalisable, permet donc le maintien du plein-emploi. Là aussi, l'accumulation des déficits courants du premier pays par rapport au deuxième amène une baisse de la demande dans le premier pays, une hausse dans le deuxième de sorte que les nouveaux prix relatifs ne sont pas tenables à terme. Au fur et à mesure que les stocks s'accumulent, il faut revenir au niveau antérieur, ce qui nécessite une forte flexibilité de la politique fiscale. De façon similaire, si le pays dominé veut réduire le poids de sa dette publique en maintenant le plein-emploi, il doit pratiquer transitoirement cette politique de baisse de ses prix relatifs.

La fixité des changes peut donc expliquer des situations combinant dans les pays dominés un fort niveau des taux d'intérêt et un fort déficit public, situations qu'ont connues la Belgique, les Pays-Bas ou la 
France après la réunification allemande. Les autres stratégies concevables pour eux auraient été soit, l'utilisation du taux de change pour stabiliser l'activité, mais cela était difficilement compatible avec le fonctionnement du SME, soit l'acceptation de la baisse du pouvoir d'achat des salaires.

Dans quelle mesure un pays peut-il respecter les critères de Maastricht à savoir certaines normes pour le déficit public et la dette publique, la fixité des changes et un taux d'intérêt imposé de l'extérieur? En économie fermée, se donner une contrainte sur la politique budgétaire, $g^{d}$, revient en fait, à plein emploi maintenu, à se donner une contrainte sur le taux d'intérêt. Comme $\mathrm{y}=\mathrm{g}+\mathrm{d}+c \mathrm{c}-\sigma_{\mathrm{r}}$, il faut, à production maintenue, que $r=\frac{g^{d}+d}{\sigma}$. Deux cas de figures peuvent se présenter alors. Soit le taux nécessaire est au-dessus du niveau plancher du taux d'intérêt réel et dans ce cas, la contrainte est accessible. Soit il est au-dessous et dans ce cas, la contrainte budgétaire est inaccessible, du moins si on veut maintenir le niveau de production. En économie ouvertee, en change fixe, le taux d'intérêt pour un pays dominé est imposé de l'extérieur. Son seul degré de liberté est sa politique budgétaire. Aussi, un taux d'intérêt trop élevé peur empêcher certains pays de réaliser les normes tequises de politique budgétaire. À cela, deux remèdes sont concevables : soit l'Allemagne baisse fortement son taux d'intérêt; chaque pays est alors en une meilleure situation pour atteindre son objectif budgétaire. Soit, les pays à fort déficit budgétaire dévaluent (ou pratiquent des politiques salariales restrictives) et pratiquent des politiques budgétaires restrictives de sorte qu'ils remplacent le déficit public par un excédent extérieur.

\section{Les changes flexibles: d'autres marges de manouvre}

On peut contraster rapidement les résultats obtenus en change fixe avec ceux que l'on obtiendrait en change flexible. Chaque pays fixe librement son taux d'intérêt. En supposant que les agents anticipent que le taux de change retournera à sa valeur de long terme, $s=0$, à une certaine vitesse $\lambda$, la relation d'équilibre sur le marché des changes $\$$ 'écrit :

\section{(8) $\mathrm{r}^{*}=r+\theta f+\lambda s$}

Sous l'hypothèse de rigidité à court terme des salaires nominaux (et donc des prix), les deux pays peuvent maintenir le plein emploi et l'équilibre budgétaire face à des chocs de demande, $\mathrm{d}$ et $\mathrm{d}^{*}$; il faut pour cela que chacun fixe son taux d'intérêt selon : $\mathrm{r}=\frac{(\mathrm{n} \delta+\sigma \lambda) \mathrm{d}+\mathrm{n} \delta \mathrm{d}^{*}}{\sigma(2 \mathrm{n} \delta+\sigma \lambda)}$; le 
taux de change variera alors selon : $s=-\frac{\left(\mathrm{d}-\mathrm{d}^{*}\right)}{2 \mathrm{n} \delta+\sigma \lambda}$. L'urilisation active de la politique monétaire permet d'éviter le creusement du déficit budgétaire et permet l'isolement des conjonctures; elle se paye toutefois de fortes fluctuations du taux de change. En sens inverse, chercher à imposer la rigidité du taux de change rend impossible la stabilisation macroéconomique en cas de choc asymétrique.

Supposons que les deux politiques budgétaires sont contraintes. Le pays qui connaît une forte conjoncture (choc $\mathrm{A}$ ) augmente son taux d'intérêt, er son taux de change s'apprécie. Son partenaire peut dès lors maintenir son activité par une hausse plus faible de son taux d'intérêt compensée par une dépréciation de son taux de change. À court terme, les deux pays maintiennent leur objectif de plein emploi. Si le choc de demande est permanent, le pays à forte demande ex ante voit son déficit extérieur se creuser. Il va accumuler une dette extérieure qui va faire baisser sa demande. Son taux de change va revenir à son niveau initial quand l'accumulation de dette étrangère sera de $f=\frac{-d}{2 k+\theta \sigma}$. La hausse du taux d'intérêt est de $\mathrm{r}=\frac{\mathrm{k}+\theta \sigma}{2 \mathrm{k}+\theta \sigma} \mathrm{d}$ chez lui; la baisse du taux d'intérêt est de $r^{*}=\frac{-\theta \sigma}{2 k+\theta \sigma} d^{*}$ chez son parrenaire. On obtient le même long terme qu'en change fixe, mais la flexibilité des changes permet d'éviter que la période transitoire se paye en chômage dans le pays dominé.

De même, un pays qui estime avoir une dette publique trop importante peut réduire cette dette tout en sauvegardant l'emploi, en combinant une politique budgétaire restrictive et une baisse de son taux d'intérêt, donc une dépréciation de son taux de change. Il est donc plus facile de réduire sa dette publique en régime de change flexible qu'en situation de pays dominé en SME; il faut néanmoins réussir à imposer une certaine discipline salariale, c'est-à-dire évirer la répercussion de la dévaluarion dans les salaires.

\section{L'organisation de l'UEM}

Nous reprenons le même modèle pour discuter de l'organisarion de la politique économique en UEM. Nous supposons qu'il n'y a pas de risque de défaut de la part d'un des deux pays. Les taux d'intérêt sont donc égaux dans les deux pays, fixés par la Banque centrale en foncrion de la moyenne de la situation économique dans l'Union. Supposons que la situation initiale représente un équilibre optimal : la demande dans les deux pays correspond au taux de chômage d'équilibre, le taux 
d'intérêt correspond au niveau optimal, les stocks d'actifs sont fixes. Cela impose a priori des niveaux de dettes publiques dans chacun des pays tels que $d+k b=0$, niveaux qui peuvent différer d'un pays à l'autre et être supérieurs à une norme prétablie si par exemple, dans un pays, les ménages souhaitent détenir une forte quantité d'actifs financiers tandis que les entreprises répugnent à s'endetter : il n'y a pas là a priori motif à remettre en cause l'équilibre macroéconomique.

En cas de choc de demande asymétrique, une stabilisation parfaite est possible si le pays touché utilise la politique budgétaire. La situation où aucun pays ne peut réagir par la politique budgétaire est moins favorable. La BCE accroît le taux d'intérêt mais moitié moins que précédemment. Elle ne résorbe donc que la moitié du choc, c'est-à-dire la moyenne sur les deux pays du choc de demande spécifique. Le plein emploi " moyen " est maintenu, mais le premier pays est en suremploi, tandis que le second est en sous-emploi. Ces déséquilibres sur le marché des biens prennent fin à long terme : le premier pays accumule des déficits et de la dette extérieurs; l'écart entre les conjonctures des deux pays s'estompent progressivement, jusqu'à ce que l'un et l'autre retrouvent leur taux de chômage d'équilibre. L'absence de marge de manœuvre budgétaire n'est donc pas compatible avec une gestion optimale des chocs spécifiques à court terme. En fait, la situation est dissyImétrique selon le sens du choc. Si le pays A subit un choc de demande positif, il peut toujours pratiquer une politique budgétaire restrictive; s'il s'y refuse, le pays B subit une hausse du taux d'intérêt, donc une chute de l'activité et des recettes fiscales. S'il subit une contrainte de déficit public, il ne peut utiliser la politique budgétaire. Si le pays A subit un choc négatif et que sa politique budgétaire est contrainte, le pays $B$ subit une baisse du taux d'intérêt, il peut donc pratiquer une politique budgétaire restrictive. À la limite, le plein-emploi peut être maintenu dans les deux pays grâce à une baisse des taux d'intérêt, mais le pays $B$ doit, en quelque sorte, se caler sur la situation du pays A par une baisse de ces dépenses publiques.

Imaginons maintenant que le pays étoilé ait un niveau des prix, $\mathrm{p}^{*}$, trop fort, mais que la demande globale soit à un niveau satisfaisant en Europe. La Banque centrale, qui ne s'intéresse qu'au niveau de la demande globale, n'a aucune raison d'agir. Le pays étoilé peut maintenir le plein emploi en ayant un déficit public $: \mathrm{g}^{*}=\mathrm{n} \delta \mathrm{p}^{*}$, égal à son déficit extérieur, tandis que son partenaire maintient le plein emploi avec un excédent public : $\mathrm{g}=-\mathrm{n} \delta \mathrm{p}^{*}$, égal à son excédent extérieur. Au cours du temps, le pays étoilé accumule une dette publique et un déficit extérieur, dont les effets sur l'activité se compensent. Aucun mécanisme de régulation n'existe alors. Pour éviter cette dérive dans les 
dépenses publiques, il faudrait soit que le pays étoilé s'engage dans une phase transitoire de sous-emploi tandis que l'autre s'engagerait dans une phase transitoire de sur-emploi de façon à rétablir une compétitivité correcte entre eux deux; soit que les deux pays mènent une politique budgétaire aboutissant à une baisse des prix dans le pays étoilé (hausse des impôts sur les ménages, baisse des impôts sur les entreprises) et l'inverse dans le pays non éroilé. Dans ce cas, la politique budgétaire doit compenser l'absence de possibilité de faire varier le taux de change.

Enfin, imaginons que le pays étoilé veuille maintenir, par la politique budgétaire, un certain niveau d'acrivité, $\mathrm{y}^{*}$, tandis que les autorités monétaires veulent stabiliser la demande globale à $y+y^{*}=0$. Il en résulte un équilibre où $y=-y^{*}$ et $r=\frac{1}{\sigma}(2 n+1-c) y^{*}$. Le surcroit de demande du pays étoilé est payé par son partenaire. On se trouve alors dans une situation conflictuelle; le second pays pouvant accuser son partenaire d'être responsable d'un niveau trop élevé des taux d'intérêt et le pays éroilé pouvant mettre en cause la Banque centrale coupable de ne pas prendre en compte correctement sa situation macroéconomique. L'UEM suppose donc un accord précis entre la Banque centrale européenne et chaque pays sur les objectifs de la politique macroéconomique dans chaque pays.

En UEM, chaque pays doit donc pouvoir bénéficier de la liberté de faire la politique budgétaire de son choix, tant que celle-ci ne met pas en cause l'équilibre macroéconomique de la zone. En la matière, la règle devrait être que, à taux d'intérêt imposé par la $\mathrm{BCE}$, un pays puisse choisir sa politique budgétaire tant que son taux d'inflation reste en ligne avec le taux d'inflation objectif de la zone er qu'il ne contribue pas de manière excessive au déficit extérieur de la Communauté. Dans ce cas, resterait néanmoins à savoir si ce pays a une politique trop expansionniste ou ses partenaires des politiques trop restrictives. Nous voyons mal comment seront évitées de telles situations conflictuelles, notamment lorsque les pays ont des objectifs différents de politique économique. Un processus de négociation er de coordination est donc nécessaire au sein de l'union. Il n'est pas prévu par le traité d'Amsterdam.

Certains économistes justifient l'existence de critères absolus de finances publiques par le risque qu'un pays se lance dans une politique budgétaire expansionniste. Ceci induirait des externalités incompatibles avec la bonne marche de la monnaie unique. Ce raisonnement suppose que les déficits publics sont toujours des déficits autonomes au sens défini plus haut : les gouvernements pratiqueraient des hausses de 
dépenses publiques ou des baisses d'impôt alors que l'économie est au plein emploi. Que de tels déficits soient nuisibles ne fait guère de doute. Il n'en reste pas moins qu'il faut distinguer a priori déficit autonome et déficit de régulation. En cas de choc de demande dépressif dans un pays, son déficit public a des vertus stabilisatrices. Le caractère indifférencié et absolu des critères de finances publiques n'est donc pas justifiable.

\section{En cas de choc symétrique}

Le cas d'un choc de demande symétrique est beaucoup plus simple à gérer. Il y apparaît que le régime de change revêt beaucoup moins d'importance qu'en cas de choc spécifique, voire pas d'importance du tout : si les deux gouvernements ne veulent pas utiliser la politique budgétaire, la politique monétaire doit intervenir; et son intervention prendra la même forme dans les trois régimes $\left(\mathrm{r}=\frac{\Sigma \mathrm{d}}{2 \sigma}\right)$. L'avantage de l'UEM réside dans sa capacité à exclure des comportements non coopératifs en terme de politique de change (dévaluations compétitives...). Toutefois, le maintien du plein-emploi dans les deux pays suppose que : $\mathrm{g}+\mathrm{d}-\sigma_{\mathrm{r}}=0$. Les autorités disposent a priori de 3 instruments pour 2 objectifs. Une règle d'affection doit en quelque sorte être définie. Elle suppose un accord sur les objectifs. Sinon, l'Europe se trouverait dans la situation de conflit que nous avons décrite en étudiant les conséquences de l'indépendance de la Banque centrale : la Banque centrale augmente le taux d'intérêt pour réduire le niveau d'activité tandis que les aurorités budgétaires augmentent le déficit public pour obrenir un niveau d'activité plus élevé. Ceci aboutirait à un équilibre non satisfaisant, marqué par un déficit budgétaire trop fort et un taux d'intérêt trop élevé, qui nuit à l'accumulation du capital.

L'instauration d'une contrainte budgétaire pour les deux pays en UEM peut ainsi sembler nécessaire puisqu'elle permet autoritairement de résoudre le conflit, par absence de négociation. Mais, elle ne règle pas la question des chocs asymétriques; elle suppose que l'Europe confie la régulation conjoncturelle à la Banque centrale; elle est inefficace en cas de choc de demande positif (car la stabilisation par la politique budgétaire est préférable dans ce cas à une stabilisation par la politique monétaire). Aussi l'instauration de critères absolus de finances publiques estelle moins importante que l'existence d'une coordination efficace entre les autorités budgétaires et monétaires. 
DU BON USAGE DES POLITIQUES MONÉTAIRE ET BUDGÉTAIRE EN EUROPE

\section{DES CRITERES DE FINANCES PUBLIQUES}

Les critères de finances publiques du traité ne se justifient donc pas du point de vue de la logique macroéconomique interne des différents pays, une fois reconnue que des déficits de régulation sont parfois nécessaires et que leur taille optimale est indéterminée a priori. Ces critères masquent le vrai problème : pour être efficace, l'UEM nécessite une forte coordination entre les politiques budgétaires nationales d'une part, et entre celles-ci et la politique monétaire d'autre part.

Dans l'UEM réalisée, les pays-membres n'auront plus à leur disposition les armes du taux d'intérêt et du taux de change, mais n'auront plus à se soucier directement de leur solde extérieur, ni à craindre qu'une politique trop expansionniste ne les oblige à monter leur taux d'intérêt, du fair d'anticipations défavorables des marchés financiers. Il y a done là un degré de liberté utile mais aussi un risque potentiel que chaque pays pratique une politique trop expansionniste faisant supporter à l'ensemble de l'Union un déficit extérieur trop fort et des taux d'intérêt trop élevés. Par ailleurs, il peut être utile dans certaines situations conjoncturelles de baisser les taux d'intérêt en Europe, quitte pour certains pays à pratiquer simultanément des politiques budgétaires plus restrictives. De telles stratégies doivent êrre décidées au coup par coup, en tenant compre finement de la situation conjoncturelle en Europe et dans chaque pays. Elles peuvent poser des problèmes délicats de négociations et de confiance réciproque. Dans son état actuel, le rrairé, s'il met en place des instances de coordination (notamment au sein du Conseil, selon l'article 103), ne prévoit guère les modalités, les principes et les objectifs de la coordination des poliriques économiques entre la $\mathrm{BCE}$ responsable de la politique monéraire et les seize détenteurs de pouvoir budgétaire (les quinze gouvernements er la CE elle-même), l'indépendance de la première et le refus des déficits publics excéssifs étant posés en principe.

La conférence inter-gouvernementale d'Amsterdam a adopté un "Pacte de stabilité " qui prolonge le plafond de $3 \%$ pour les déficits publics, sans organiser la coordination des poliriques économiques en Europe. En cas de dépassement de la limire au déficit public, la Pacte précise les délais de réaction et le rôle des différentes institutions communautaires dans la procédure de sanctions, ainsi que le montant de celles-ci. Heureusement, la norme de dette publique n'est plus évoquée explicitement. Le Pacte n'est envisageable que dans une siruation où, face à un choc symétrique négatif, la politique monétaire de l'Union est fortement assouplie. Ainsi, pour être applicable, il nécessite une forte coordination entre la Banque centrale européenne et les autorités bud- 
gétaires de tous les États membres, ce qui parait peu compatible avec Pindépendance de la BCE. Face à un choc asymétrique, l'augmentation du déficit public dans le pays touché est inéluctable.

Se refusant à organiser la coordination des politiques budgétaires entre elles et la coordination des politiques budgétaires avec la politique monétaire, le traité d'Amsterdam met en place des barrières immuables et indifférenciées aux déficits publics, sans tenir compte dans le détail des considérations, conjoncturelles ou structurelles, qui peuvent rendre souhaitable un franchissement durable de ces barrières. On voit mal comment seront évitées des situations conflictuelles.

Aussi, faut-il oublier les critères de finances publiques. Par contre, il convient de s'interroger sur les règles de conduite de la politique économique en situation de monnaie unique, de façon à éviter soit des crirères automatiques inadéquars, soit des situations conflictuelles entre les différentes autorités, nationales ou communautaires.

\section{REFFERENCES BIBLIOGRAPHIQUES}

AgLietTA M, V. COUderC et B. Mojon (1993) : « Déflation financière : les leçons de l'histoire ", Lettre du CEPII, mars.

Begg D., P.-A. Chiapport, F. Glavazzi, C. Mayer, D. Neven, L. Spaventa, X. VIVES, et C. WYPLOSZ (1991), Monitoring European Integration, the making of Monetary Union, CEPR.

Bismut C. et J. PISANd-Ferry (1994), "L'Union économique et monétaire, prinicipes et implications ", Revue économique, hors-série.

Bovenberg A.-L., J.J.-M. Kremers et P.-R. MASSON (1991), "Economic and Monetary Union in Europe and Constraints on National Budgetary Policies", IMF Staff Papers, vol.38, n² 2 , juin.

Branson W.H. (1976), "Assets Markets and Relative Prices in Exchange Rate Determination ", Seminar Paper, $n^{\circ}$ 66, Srockholm Institute for International Economic Studies, décembre.

BuITER W.H. (1992), "Should We Worry about the Fiscal Numerology of | Maastricht? ", CEPR Discussion Paper Series $n^{\circ} 668$, juin.

Butter W.H., G. Corsetti et N. Roubini (1993), "Excessive Deficits : Sense and Nonsense in the Treary of Maastricht ", Economic Policy, n 16.

BUITER W.H. et K.N. KIETZER (1991), " Reflections on the Fiscal Implications of a Common Currency ", dans A. GIovanNins et C. Mayer (éds.), European Financial Integration, CEPR, Cambridge University Press.

Cadiou L, B. Coquet, H. Le Bihan, F. Lerais et H. Sterdyniak (1997) : "UEM : Quelle stratégie macroćconomique?", Miméo, colloque de l'AEA, Rome, novembre. 
Capoén F., H. Sterdiniak et P. Villa (1994), "Indépendance des Banques Centrales, Politiques Monétaire et Budgétaire : une Approche Stratégique », Revue de l'OFCE, $\mathrm{n}^{\circ} 50$, juiller.

CHRist C. (1968), "A Simple Macroeconomic Model with a Government Budger Constraint ", Journal of Political Economy, vol. 76.

Creel J. et H. Sterdyniak (1995), "Les Déficits Publics en Europe : Causes, Conséquences ou Remèdes à la Crise? ", Revue de l'OFCE, $\mathrm{n}^{\circ} 54$, juillet.

Delessy H., F. Lerais, S. Paris-Horvitz et H. Sterdyniak (1993), "Après Maastricht: Quelles Politiques Économiques en Europe?" Revue de l'OFCE, $n^{\circ} 43$, janvier.

Giavazzi F. et M. Pagano (1990), "Can Severe Fiscal Contractions Be Expansionary? Tales of Two Small European Councries ", dans BLANCHARD O.-J. et S. FISCHER (éds.), NBER Macroeconomics Annual, The MIT Press.

Glovannini A. ex L. Spaventa (1991), "Fiscal Rules in the European Monetary Union : a No-entry Clause ", CEPR Discussion Paper Series n 516 , janvier.

DE Grauwe P. (1994), The Economics of Monetary Integration, Oxford University Press.

De Grauwe P. (1996), "The Economics of Convergence : Towards Monetary Union in Europe ", Weltwirtschaftiches Archits, vol.132, $\mathrm{n}^{\circ} 1$.

Gros D. (1995), "Towards a Credible Excessive Deficits Procedure ", Working Document, Centre for European Policy Studies, Brussels.

KENEN P.B. (1991), EMU after Maastricht, Group of Thirry, Washingcon D.C.

Le Bihan H, Sterdynlak H. et Ph. Cour (1997) : "La nocion de croissance potentielle a-t-elle un sens? ", Economie internationale, I er trimestre.

LORDON F. (1994) : "Marchés financiers, crédibilité er souveraineré ", Observations et Diagnostics économiques, juiller.

Mundell R. (1993) : "Rejection of the Common Funnel Theorem ", Rivista di Politica Economica, décembre.

Nordhaus W. (1993) : “Coordination and Independence in Monetary and Fiscal Policy ", Rivista di Politica Economica, décembre.

PHeLPS E.S. (1994) : Structural Slumps : the Modern Equilibrium Theory of Unemployment, Interest and Assets, Harvard Universicy Press.

Rapporr DeLors (1989), Rapport sur l'Union économique et monétaire dans la Communauté européenne, Bureau des publications officielles de la Communauté européenne, Luxembourg, avril.

Sterdiniak H. et P. Villa (1993), "Régines de Change et Coordination des Politiques économiques en Europe ", Revue de l'OFCE, n 43 , janvier.

WypLOSZ C. (1990), "Les Implications Budgétaires de l'Union monétaire ", Revue de l'OFCE, $\mathrm{n}^{\circ} 33$, octobre. 
REVUE D'ECONOMIE FINANCIERE

NOTES

1. Voir Rapport économique annuel 1997, p. 85.

2. Au niveau de généraalité où nous nous plaçons ici, $\mathrm{g}$ peur représenter les dépenses publiques en biens, les transferts aux ménages ou les réductions d'impôts portant sur leṣ ménagess. Nous n’introduisons pas 4ans l'analyse la possibiliré d'engager une politique budgécaire expansionniste par hausse des subventions aux entreprises, réduction des charges portanr sur elles ou baisse des impôrs indirects.

\$. Ceci au nom du principe d'affectarion de MundonL (1963), selon lequel : " Il faut affecter chaque instrument de politique économiquc à l'objectif qu'il influence lc plus relativement. *

1. On trouvera une discussion sur ce poinr dans les artickes de Nordhaus (1993) et MUNDELl (1993). Tourefois, aucun de ces articles ne prend en compre l'impact spécifique de la politique monétaire sur lia sicuation des entreprises. Mundell fait l'éloge de la politique de surévaluarion du change, comme arme anti-inflationniste, sans discuter ni de son impacr sur les pays partenaires, ni de son effet sur la łompéritivié, donc sur la balance commerciale. 